\title{
Die SAMW lehnt die vorgeschlagene Neu- regelung der organisierten Suizidbeihilfe ab
}

\author{
Schweizerische Akademie \\ der Medizinischen \\ Wissenschaften (SAMW)
}

1 Rietjens JA et al. A comparison of attitudes towards end-of-life decisions: survey among the Dutch general public and physicians. Soc Sci Med. 2005;61(8):1723-32.

2 Vollmann J, Herrmann E. Attitudes of psychiatrists toward physician-assisted suicide, Fortschr Neurol Psychiatr. 2002;70(11):601-8.

Korrespondenz: SAMW

Petersplatz 13

CH-4051 Basel

mail@samw.ch
Der Bundesrat will die organisierte Sterbehilfe regeln und hat Ende Oktober 2009 zwei Varianten in die Vernehmlassung geschickt. Die vom Bundesrat bevorzugte Variante 1 sieht die Einführung strenger Sorgfaltspflichten vor. Neu sollen im Strafgesetzbuch Art. 115 Abs. 2 sieben Voraussetzungen eingefügt werden, die ein Suizidhelfer, der im Rahmen einer Organisation handelt, einzuhalten hat:

- Der Entscheid der suizidwilligen Person ist frei gefasst, wohlerwogen und dauerhaft.

- Die sterbewillige Person ist urteilsfähig, was durch einen unabhängigen Arzt zu prüfen ist.

- Die Person ist unheilbar krank mit unmittelbar bevorstehender Todesfolge, was durch einen zweiten unabhängigen Arzt festzustellen ist.

- Andere Hilfestellungen als der Suizid wurden erörtert und auch angeboten.

- Der Suizid wird mit einem ärztlich verschriebenen Mittel durchgeführt.

- Der Helfer verfolgt keinen Erwerbszweck.

- Die Organisation und der Helfer erstellen eine Dokumentation.

Variante 2 des Vorschlags sieht ein generelles Verbot der organisierten Suizidbeihilfe vor.

Die Schweizerische Akademie der Medizinischen Wissenschaften (SAMW) hat sich eingehend mit der vorgeschlagenen Neuregelung auseinandergesetzt und dem Bundesrat die nachfolgende Stellungnahme unterbreitet.

\section{Die vorgeschlagene Regelung der organisierten Suizidbeihilfe löst die bestehenden Probleme nicht}

Die Schweizerische Akademie der Medizinischen Wissenschaften (SAMW) setzt sich seit Jahrzehnten intensiv mit Fragen der Sterbehilfe und Sterbebegleitung auseinander. Ihre medizinisch-ethischen Richtlinien setzen in diesem Bereich Standards für das medizinische Handeln. Die SAMW hat dabei immer vertreten, dass im Bereich der indirekt aktiven und der passiven Sterbehilfe keine gesetzlichen Regelungen notwendig sind, sondern die Richtlinien das geeignete Instrument sind. Im Gegensatz dazu setzt sich die SAMW jedoch seit längerem für eine Aufsicht über die Tätigkeit der Sterbehilfeorganisationen ein. In einem Schreiben an den Bundesrat vom 7. Juni 2006 hat sie dies wie folgt begründet:

"Der Schutz einer sterbewilligen Person ist aus unserer Sicht absolut entscheidend. (...) Dass gerade da, wo es um Leben und Tod geht, nur ungenügende Sorgfaltskriterien und keine Aufsicht bestehen, ist unbefriedigend. (...) Wir sind klar der Auffassung, dass der Bund im Bereich der Sterbehilfeorganisationen eine Aufsichtspflicht hat.»

Vor diesem Hintergrund begrüsst die SAMW die Bemühungen des Bundes zur Regelung der organisier- ten Suizidbeihilfe grundsätzlich. Sie unterstützt insbesondere die Tatsache, dass lediglich ein Teil der Suizidhilfepraxis, nämlich die organisierte Beihilfe, geregelt werden soll. Aus Sicht der SAMW erscheinen jedoch weder die vorgeschlagene Beschränkung der organisierten Suizidbeihilfe (Variante 1) noch deren Verbot (Variante 2) - über eine Regelung im Strafgesetzbuch, respektive im Militärstrafgesetz - ein geeigneter Weg zur Lösung der aktuellen Probleme.

Die SAMW lehnt die vorgeschlagene Regelung aus den folgenden Gründen ab:

- Die Suizidbeihilfe wird als ärztliche Tätigkeit institutionalisiert.

- Der Druck auf medizinische Institutionen, Sterbehilfeorganisationen in ihren Räumlichkeiten zuzulassen, wird erhöht.

- Die Beschränkung auf das «unmittelbare Lebensende» ist untauglich.

- Die vorgeschlagene Regelung ist leicht zu umgehen.

\section{Die Suizidbeihilfe wird als ärztliche Tätigkeit institutionalisiert}

Mit der vorgeschlagenen Neuregelung würden Ärztinnen und Ärzte stärker als bisher in die Suizidbeihilfe involviert. Einerseits werden ihnen in der Variante 1 drei zentrale Aufgaben übertragen (Feststellung des Lebensendes, Abklärung der Urteilsfähigkeit sowie Verschreibung des NAP), die den Druck auf den einzelnen Arzt erhöhen, Suizidbeihilfe zu leisten bzw. Aufgaben zu übernehmen, die im Zusammenhang mit einem geplanten Suizid stehen. Andererseits wird die Beschränkung der vorgeschlagenen Regelung in den Varianten 1 und 2 auf die organisierte Suizidbeihilfe dazu führen, dass chronisch kranke Sterbewillige, die sich nicht unmittelbar am Lebensende befinden, sich mit ihrem Wunsch vermehrt an Ärzte wenden werden. Erstaunlicherweise werden diese Auswirkungen auf die Rolle des Arztes im erläuternden Bericht in keiner Weise thematisiert.

Die SAMW-Richtlinien «Betreuung von Patientinnen und Patienten am Lebensende» tolerieren die ärztliche Suizidbeihilfe im Einzelfall und innerhalb eines gewissen Rahmens als persönliche Gewissensentscheidung eines Arztes. Die SAMW hat jedoch mehrfach festgehalten, dass die zunehmende Etablierung der Suizidbeihilfe in der Verantwortung der Gesellschaft als Ganzes liege und nicht an die Ärzteschaft delegiert werden könne. Wie der einzelne Arzt mit dem aktuell vom Recht eingeräumten Handlungsspielraum umgeht, hängt heute von seinem Gewissen ab. Es ist empirisch belegt, dass die Akzeptanz der Suizidbeihilfe in der Bevölkerung grösser ist als in der Ärzteschaft [1] und innerhalb dieser umso geringer, je mehr Erfahrung der betreffende Arzt mit der Betreuung von Patienten am Lebensende hat [2]. 
Auch internationale Erfahrungen lassen vermuten, dass hier ein Konfliktpotential besteht, das eng mit dem Selbstverständnis ärztlicher Tätigkeit zusammenhängt: Ärztinnen und Ärzte sehen eine wichtige Aufgabe darin, einen leidenden Menschen am Lebensende zu begleiten und auch in schwierigen Situationen nicht im Stich zu lassen; hingegen betrachten sie es nicht als ihre Aufgabe, Menschen, die aus unterschiedlichen Gründen wie Vereinsamung, Isolation, Behinderung oder psychische Erkrankung schwer leiden, sich aber nicht am Lebensende befinden, bei einem Suizid zu unterstützen. Aktuell liegt heute in über 30\% der Fälle von organisierter Suizidbeihilfe keine Situation am Lebensende vor [3]. Es ist deshalb wahrscheinlich, dass mit den vorgeschlagenen Neuregelungen (Variante 1 und 2) der Druck auf den einzelnen Arzt, Suizidbeihilfe zu leisten, steigen wird.

Zum stärkeren Einbezug der Ärzteschaft in die Suizidbeihilfe trägt auch die in Variante 1 vorgesehene Regelung bei, die Feststellung der Urteilsfähigkeit ausschliesslich in die ärztliche Verantwortung zu legen. Aus Sicht der SAMW muss eine Zweitmeinung nur dann zwingend durch einen Arzt oder eine Ärztin erfolgen, wenn es Unsicherheiten in medizinischen Fragen gibt (z. B. bei der Abklärung der Urteilsfähigkeit von Patienten mit beginnender Demenz oder Patienten mit einer psychischen Störung).

\section{Der Druck auf medizinische Institutionen,} Sterbehilfeorganisationen in ihren Räumlichkeiten zuzulassen, wird erhöht

Viele Menschen verbringen ihre letzte Lebensphase im Spital oder in einem medizinisch betreuten Lebensmilieu (Pflegeheim). Mit der in Variante 1 gewählten Einschränkung der organisierten Suizidbeihilfe auf das unmittelbare Lebensende würde somit der Druck auf Spitäler und Pflegeheime steigen, Sterbehilfeorganisationen in ihren Räumlichkeiten zuzulassen. Die Suizidbeihilfe steht jedoch im Widerspruch zum primären Auftrag dieser Institutionen. Würden sie deshalb weiterhin mehrheitlich an ihrer Praxis festhalten, Sterbehilfeorganisationen den $\mathrm{Zu}$ tritt zu verweigern, käme dies aber faktisch einem Verbot der organisierten Suizidbeihilfe (Variante 2) gleich, was auch vom Bundesrat nicht favorisiert wird.

\section{Die Beschränkung der Regelung auf das} «unmittelbare Lebensende» ist untauglich Die SAMW hat in ihren Richtlinien das Kriterium «Lebensende» eingeführt, um Ärztinnen und Ärzten, die in der Betreuung von Sterbenden involviert sind, Hilfe zu leisten in Grenzsituationen, in denen sie mit einem Suizidwunsch konfrontiert sind. Als justiziables Kriterium zur Regelung der organisierten Suizidbeihilfe ist das «Lebensende» aber kaum geeignet. Einerseits bestehen bekanntermassen Schwierigkeiten bei dessen Prognose, andererseits widerspricht eine solche Beschränkung der Einstellung und der Erwartung weiter Teile der Bevölkerung.

\section{Die vorgeschlagene Regelung ist leicht zu umgehen}

Die vorgeschlagenen Varianten lösen die Probleme der heutigen Situation letztlich nicht, da die Einschränkungen leicht umgangen werden können: Die Voraussetzungen müssen nur von Organisationen erfüllt werden, nicht von Einzelpersonen oder Ärzten. Damit kann die Organisation Sterbewillige, welche die Voraussetzungen nicht erfüllen, an zugewandte Ärzte vermitteln, die nicht durch die neue Regelung gebunden sind.

\section{Vorschläge zur Regelung mit einem Spezialgesetz}

In seinem Bericht über «Sterbehilfe und Palliativmedizin - Handlungsbedarf für den Bund?» vom 24. April 2006 hatte das Eidgenössische Justiz- und Polizeidepartement verschiedene Möglichkeiten zur Verhinderung von Missständen im Bereich der Suizidbeihilfe in Erwägung gezogen. Neben einer Revision des Art. 115 Strafgesetzbuch hat das EJPD auch eine Regelung mittels Aufsichtsgesetzgebung geprüft. Gestützt auf die abschliessende Empfehlung des Berichts hatte der Bundesrat im Mai 2006 noch auf einen Vorschlag für eine Regelung verzichtet.

Seitdem haben sowohl parlamentarische Vorstösse (z.B. Motionen Nr. 073163 und 07.3626) als auch die Nationale Ethikkommission im Bereich der Humanmedizin (NEK) und die SAMW vom Bundesrat die Schaffung einer gesetzlichen Grundlage für die Aufsicht über Sterbehilfeorganisationen verlangt; dennoch wird die Möglichkeit einer Aufsichtsregelung im Begleitbericht zur vorgeschlagenen Revision des Strafgesetzbuches in Ziff. 4.3. nur ganz kurz behandelt. Der Verzicht auf eine Regelung mittels Spezialgesetz wird damit begründet, dass damit eine gewisse Bürokratisierung der organisierten Suizidbeihilfe unumgänglich würde. Diese Argumentation leuchtet jedoch nicht ein. Aus Sicht der SAMW wäre eine Aufsichtsgesetzgebung zur organisierten Suizidbeihilfe insofern eine geeignete Lösung, weil damit einerseits Suizidbeihilfeorganisationen einer Bewilligungspflicht unterstellt und andererseits die Einhaltung bestimmter Sorgfaltskriterien überprüft werden könnten.

In einem Aufsichtsgesetz sollten folgende Punkte geregelt werden:

- Sorgfaltspflichten für Suizidhelfer, insbesondere sorgfältige Auswahl der Personen, adäquate Ausbildung sowie Supervision;

- Sorgfaltspflichten für Ärztinnen und Ärzte, welche mit Organisationen der Suizidbeihilfe zusammenarbeiten, insbesondere für Ärzte, die das eingesetzte Medikament verschreiben;

- Transparenz bzgl. Geschäftsführung und Statistik.

\section{Fazit}

Gestützt auf die vorstehenden Ausführungen lehnt die SAMW die vorgeschlagenen Regelungen betreffend die organisierte Suizidbeihilfe ab und schlägt eine Regelung mittels Aufsichtsgesetzgebung für Sterbehilfeorganisationen vor. Sie empfiehlt dem Bund zudem, die Anstrengungen zur Suizidprävention zu verstärken und Palliative Care weiter zu fördern. 